\title{
6. COMMISSION DES TELEGRAMMES ASTRONOMIQUES
}

\author{
Président: E. Buchar. \\ Directrice DU BUREAU: Mlle J. M. Vinter Hansen. \\ Membres: MM. Cunningham, A. R. Hogg, Merton, Mikhailov, Platzeck, van den Bos, \\ Whipple, Director Harvard Observatory.
}

\section{REPORT OF THE CENTRAL BUREAU FOR ASTRONOMICAL TELEGRAMS}

The work of the Bureau since 1955 has followed the usual lines. The number of subscribers to telegrams and circulars, and to circulars only, has shown a considerable rise, namely, from 64 to 68 and from 87 to II 7 respectively. Pleasing as is this rise in the numbers of subscribers, it calls for a greater amount of clerical work at the Bureau, and with the rising of printing costs it has only been possible to keep the subscription fees fairly low because of the subsidies received from the I.A.U. and from the Danish RaskOrsted Foundation. These subsidies have up to I952 paid for all the clerical work, but are now barely able to do so, and the Bureau may have to ask for a somewhat larger subsidy from the I.A.U. We try as far as possible to satisfy the various wishes of our subscribers, but it has to be repeated that the work of our secretary is often made unnecessarily complicated and burdensome when orders for telegrams and circulars reach us through book agencies. The Bureau would greatly appreciate if subscribers would deal direct with the Bureau as far as possible; such direct subscriptions also are cheaper to the subscribers.

As to the more scientific work involved in the activities of the Bureau we have been greatly helped by astronomers not attached to the Copenhagen Observatory, primarily by the B.A.A. computers, but also by many others, for instance in Czechoslovakia, U.S.A., and the U.S.S.R.

An unexpected and rather hectic activity at the Bureau arose from the launching of the first Soviet artificial satellite. This impressive Soviet achievement sent all our telephones ringing day and night, and furthermore Dr Whipple of the Smithsonian Astrophysical Observatory asked us to act as a clearing centre for European observations. $\mathrm{Mr}$ K. A. Thernöe, first assistant at the Copenhagen Observatory, volunteered to sift all incoming material, a not inconsiderable work, as many of the data came from laymen not accustomed to observing. Presumably more satellites will be launched in the near future, both from the U.S.S.R. and from the U.S.A., and we realize that the staff of the Copenhagen Observatory and that of the Bureau, as organized at present, is much too small to take on satellite work of such dimensions. It would therefore seem advisable that the I.A.U. at the Moscow congress, if not earlier, discuss whether a new International Bureau for such satellite work should be established under the auspices of the I.A.U.

Below are given the number of telegrams received and transmitted to the subscribers together with the number of circulars issued, all in the period since the report in Trans. I.A.U. vol. 9, that is from 20 October I954 until 20 October 1957. Finally a list is given of present subscribers to the services of the Bureau:

\begin{tabular}{|c|c|c|c|}
\hline & \multicolumn{2}{|c|}{ Telegrams } & \multirow[b]{2}{*}{ Circulars } \\
\hline & Received & Sent & \\
\hline 1954 (from 20 Oct.) & 3 & 58 & $7(1479-1485)$ \\
\hline 1955 & 32 & 363 & 49 (1486-1534) \\
\hline 1956 & 25 & 277 & $45(1535-1579)$ \\
\hline 1957 (until 20 Oct.) & 46 & 277 & $43(1580-1622)$ \\
\hline & 106 & $\overline{975}$ & $\overline{144}$ \\
\hline
\end{tabular}




\section{TELEGRAMMES}

\section{Subscribers to telegrams and circulars}

Harvard College Observatory, Cambridge, Mass., U.S.A.

Union Observatory, Johannesburg, South Africa.

Mount Stromlo Observatory, Canberra ACT, Australia.

Astronomitcheskij Institut im. Sternberga, Moskva 22, U.S.S.R.

Astronomisches Rechen-Institut, Heidelberg, Germany.

Royal Observatory, Edinburgh 9, Scotland.

Royal Greenwich Observatory, Hailsham, Sussex, England.

Norman Lockyer Observatory, Sidmouth, S. Devon, England.

Observatorio Fabra, Barcelona, Spain.

Observatorio astronomico, Madrid, Spain.

Observatorio de Marina, San Fernando, Spain.

Observatoire de l'Université de Bordeaux à Floirac, Gironde, France.

Observatoire, Marseille, France.

Observatoire National, Paris $\mathrm{r}_{4}$, France.

Observatoire, Strasbourg, Bas-Rhin, France.

Observatoire d'Alger, Bouzaréa, Alger, Africa.

Sterrewacht, Leiden, Holland.

Osservatorio astronomico Capodimonte, Napoli, Italy.

Osservatorio astronomico di Brera, Milano, Italy.

Osservatorio astronomico dell' Università, Padova, Italy.

Observatoire, Bucarest, Roumania.

Observatoriet, Stockholm-Saltsjöbaden, Sweden.

Observatoire, Genève, Switzerland.

Observatory, Helwan, Egypt.

Observatoire Royal, Uccle, Belgium.

Observatoire astronomique de Tokyo, Mitaka près Tokyo, Japan.

Observatoire d'Astronomie physique, Meudon, S. et O., France.

Royal Astronomical Society, Burlington House, London W. r, England.

Osservatorio astronomico, Monte Mario, Roma, Italy.

Observatory, Athens, Greece.

Osservatorio astronomico, Trieste.

Obserwatorium Uniwersitetu Poznań, Poznań 8, Poland.

Observatoriet, Uppsala, Sweden.

Observatoire Astronomique, Beograd, Yugoslavia.

Specola Vaticana, Castel Gandolfo, Roma, Italy.

Observatorio astronomico da Universidade de Coimbra, Portugal.

Observatoriet, Lund, Sweden.

Observatoriet, Yliopisto, Turku, Finland.

Observatorio Nacional, Rio de Janeiro, Brazil.

Institut Astronomique, Sofia, Bulgaria.

Ole Römer-Observatoriet, Aarhus, Denmark.

Institut d'Astrophysique, Paris I4, France.

Observatoire de Haute Provence, Saint-Michel l'Observatoire, Basses-Alpes, France.

Dr Leland Cunningham, Leuschner Observatory, Berkeley 4, California, U.S.A.

Astronomisches Institut, Bern, Switzerland.

The University of London Observatory, London N.W.7, England.

Osservatorio astronomico della Università, Bologna, Italy.

Astronomical Association, c/o Dr Merton, Oxford, England.

Dr E. Leutenegger, Frauenfeld, Switzerland.

The Observatories, Cambridge, England.

Obserwatorium, Warszawa, Poland.

Dunsink Observatory, Co. Dublin, Eire.

Carter Observatory, Wellington W. I, New Zealand. 


\section{COMMISSION 6}

Armagh Observatory, Armagh, Northern Ireland.

Konkoly Observatory, Budapest XII, Hungary.

Observatoire de la Société Astronomique de France, Paris 6, France.

Universitäts-Sternwarte, Wien I ro, Austria.

Institut d'Astronomie de l'Université de Lausanne, Switzerland.

C.S.I.R.O. (Radiophysics), University Grounds, Chippendale, N.S.W., Australia.

Dr D. J. Febrer, Barcelona, Spain.

Astronomické Observatorium, Tatranská Lomnica, Skalnaté Pleso, Czechoslovakia.

Laboratoire d'Astronomie de la Faculté des Sciences, Lille, France.

Astronomický Ústav ČSAV, Ondřejov, Czechoslovakia.

Purple Mountain Observatory, Nanking, China.

Polskie Towarzystwo Milosnikow Astronomii, Kolo Warszawa, Warszawa, Poland.

Polska Akademia Nauk, Osrodek Bibliografii i Dokumentacji Naukowej, Biblioteka, Warszawa, Poland.

Katedra Astronomii Geodezyjnej, Warszawa, Poland.

Agrupación Astronomica Aster, Barcelona, Spain.

\section{Subscribers to circulars}

Instituttet for teoretisk Astrofysikk, Blindern, Norway.

Observatoire astronomique de l'Université de Jassy, Jassy, Roumania.

Lick Observatory, Mount Hamilton, California, U.S.A.

Yerkes Observatory, Williams Bay, Wisconsin, U.S.A.

Mount Wilson Observatory, Pasadena, California, U.S.A.

U.S. Naval Observatory, Washington 25, D.C., U.S.A.

Serials Department, The General Library, University of California, Berkeley 4, California, U.S.A.

Observatoire Astronomique, Kopernika 27, Kraków, Poland.

Ananaian Institute of Astro-Computation, Shimizu-Shi, Japan.

Brown University Library, Ladd Observatory, Providence 6, R.I., U.S.A.

Observatoire de Juvisy, Seine et Oise, France.

Astronomisches Institut der Universität, Lwow, U.S.S.R.

Taschkent Astronom. Observatorium, Komitet Nauk Pri S.N.K., U.S.S.R., Fundamentaln. Biblioteka, Taschkent, U.S.S.R.

Bosscha Sterrewacht, Lembang, Java, Indonesia.

Observatory of the University of Michigan, Ann Arbor, Michigan, U.S.A.

Observatoire de Toulouse, Toulouse, Haute-Garonne, France.

Institut d'Astrophysique de l'Université de Liège, Cointe-Sclessin, Belgium.

Professor Oswald Thomas, Wien III, Austria.

H.M. Nautical Almanac Office, Royal Greenwich Observatory, Hailsham, Sussex, England.

Hans Q. Rasmusen, Værslevgaarden, Værslev, Denmark.

Nizamiah Observatory, Begumpet, Hyderabad-Deccan, India.

Glavnaja Astronom. Observatorija S.S.S.R. v Pulkove (GAO), bliz Leningrad, V.O., U.S.S.R.

The Astronomical Society of Edinburgh, r 26 West Savile Terrace, Edinburgh 9, Scotland.

Royal Observatory, Cape Town, South Africa.

Astrophysikalisches Observatorium, Potsdam, Germany.

Hamburger Sternwarte, Bergedorf, Germany.

Universitäts-Sternwarte, Potsdam-Babelsberg 2, Germany.

Sternwarte Sonneberg in Thüringen, Germany.

Kwasan Observatory, Yamashina, Kyoto, Japan.

Observatoire, Besançon, France.

J. Peridier, Observatoire Le Houga, Les Arousettes, (Gers), France.

Observatorio Astronomico, Cordoba, Argentina.

Warner and Swasey Observatory, Case Institute of Technology, East Cleveland I2, Ohio, U.S.A. 


\section{TELEGRAMMES}

Biblioteka Akademii Nauk, Leningrad, U.S.S.R.

Dr C. Luplau Janssen, Urania-Observatoriet, Copenhagen F, Denmark.

Astronomiska Observatoriet, Helsingfors, Finland.

Eidgenössische Sternwarte, Zürich, Switzerland.

McDonald Observatory, Fort Davis, Texas, U.S.A.

University of California at Los Angeles, Library, Los Angeles 24, California, U.S.A.

Lowell Observatory, Flagstaff, Arizona, U.S.A.

Astronomical Observatory of Wroclaw University, Wroclaw, Poland.

Robert A. Naef, 'Orion', Meilen (Zürich), Switzerland.

Jodrell Bank Experimental Station, Lower Withington, nr. Crewe, England.

Cincinnati Observatory, Cincinnati 8, Ohio, U.S.A.

Sterrewacht 'Sonnenborgh', Zonnenburg 2, Utrecht, Holland.

Professor Dr A. Kaufmann, Sternwarte, Solothurn, Switzerland.

Observatorio Astronomico, Montevideo, Uruguay.

The Institute for Theoretical Astronomy (Academy of Sciences of the U.S.S.R.), Leningrad, U.S.S.R.

Observatoire du Pic du Midi, Bagnères de Bigorre, Hautes Pyrénées, France.

Universitäts-Sternwarte, Jena, Germany.

Yale University Observatory, New Haven i I, Conn., U.S.A.

Osservatorio astronomico di Torino, Pino Torinese, Italy.

A. Schmuhl, Copenhagen, Denmark.

R. Rigollet, Paris I6, France.

Biblioteka Akademii Nauk, Tbilisi, U.S.S.R.

Bibliothèque du Bureau des Longitudes, Paris 6, France.

Universitäts-Sternwarte, Innsbruck, Austria.

California Institute of Technology, Astrophysics Library, Palomar, Pasadena 4, California, U.S.A.

Department of Astronomy, The University, Manchester I3, England.

La Société d'Astronomie d'Anvers, Anvers, Belgium.

J. Storms, Berchem-Anvers, Belgium.

Observatorio astronomico de la Universidad de Chile, Santiago de Chile.

Observatoire Mont Gros, Nice, France.

Filial Biblioteki Akademii Nauk S.S.S.R., Moskva 57, U.S.S.R. (two subscriptions).

University of Pennsylvania Library, Philadelphia 4, Pa., U.S.A.

Moskovskoje Otdelenije Biblioteki Akad. Nauk, Moskva 7I, U.S.S.R.

Astronomisch-Meteorologische Anstalt der Universität Basel, Binningen, Switzerland.

Amherst College, Department of Astronomy, Amherst, Mass., U.S.A.

Perkins Observatory, Delaware, Ohio, U.S.A.

Observatorio Cagigal, Caracas, Venezuela.

Ustřední Knihovna, Fakulty zeměm., Praha I, Czechoslovakia.

Osservatorio Astrofisico di Catania, Catania, Italy.

Oblastní lidová hvězdárna, Plzeñ, Czechoslovakia.

Biblioteka Akademii Nauk SSSR, Leningrad I64, U.S.S.R. (two subscriptions).

Ole Hesselberg, Næstved, Denmark.

Dr R. Leclaire, Montreal, Canada.

Dr R. L. Waterfield, Ascot, Berks., England.

Biblioteka Akademii Nauk, Tallin, U.S.S.R.

Ustredna knižnica prirodoved. fak. U.K. Bratislava, Czechoslovakia.

Urania-Sternwarte, Zürich, Switzerland.

U.S. Naval Observatory, Flagstaff, Arizona, U.S.A.

Gos. Nauk. Biblioteka, Minist Vyssh. Obraz., Moskva, U.S.S.R. (two subscriptions).

Biblioteca Academiei RPR, Bucarest, Roumania.

Landessternwarte, Königstuhl, Heidelberg, Germany.

The Library, Academia Sinica, Peking, China.

Uttar Pradesh State Observatory, Naini Tal, India. 


\section{COMMISSION 6}

Centre de Documentation du C.N.R.S., Rédaction du B ${ }^{\text {in }}$ Analytique, Paris 5, France.

Brorfelde Observatoriet, pr. Töllöse, Denmark.

Astronomický ústav CSAV, Praha 12, Czechoslovakia.

Astronom. Institut der Karls Universität, Praha I6-Smíchov, Czechoslovakia.

Lidová hvězdárna U.N.V. hl.m. Prahy, Praha IV-Petłín 205, Czechoslovakia.

Slovenska akademie vied, Bratislava, Czechoslovakia.

Observatoire Cantonal, Neuchatel, Switzerland.

Knishnij Otdel. Akad. Nauk., Moskva 34, U.S.S.R.

Guozi Shudian, P.O., Peking, China (eight subscriptions).

Sky and Telescope, Harvard College Observatory, Cambridge 38, Mass., U.S.A.

Biblioteka Uniwersytecka, Torun, Poland.

Planetarium i Obserwatorium Astronom. im. M. Kopernika, Chorzbw I, Poland.

Astronomski Institut, Univerze Ljubljana, Yugoslavia.

Observatorio Astronomico Nacional, Tacubaya 18, Mexico.

Cruset, Paris I6, France.

A. Lemans, Zürich 5I, Switzerland.

Observatorio Astrofisico de Tonantzintla, Puebla, Pue., Mexico.

Dr Rawi Bhavilai, Physics Dept., Faculty of Science, Chulalongkorn University, Bangkok,

Thailand.

Observatory, St Andrews, Scotland.

Wiener Astronom. Volksbildungsstelle der Volkhochschule, Wien 6, Austria.

Government Astronomer, Perth, Western Australia.

PResident: E. Buchar.

Report of Meeting. I5 August I958

SeCRetary: Miss J. M. Vinter Hansen.

The President reported that he had asked members of the Commission for new proposals as to the activity of the Telegram Bureau, but no such proposals had been sent to him.

Dr Cunningham had written that he did not think it advisable to include artificial satellites in the work of the Telegram Bureau; after a short discussion the Commission came to the conclusion that at present it is not possible for the Bureau to include in the circulars anything other than very reliable orbits for these objects.

In accordance with her statement in the Draft Report Miss Vinter Hansen asked the Commission to support her plea for a larger annual grant from the I.A.U., and a resolution asking for the annual grant to be raised from I 200 gold francs to I600 gold francs was sent to the Finance Committee (Resolution no. Io).

Miss Vinter Hansen asked the Commission to inspect the accounts for the preceding three years since the Dublin meeting; these accounts had been duly audited by Sir Harold Spencer Jones.

At the end of the meeting the President expressed his thanks to the Danish RaskOersted foundation for the financial aid it gives the Telegram Bureau and also his thanks to Sir Harold Spencer Jones for his kindness in agreeing to do the not inconsiderable work of auditing the accounts of the Bureau. The President also expressed his appreciation of the efficient way in which the affairs of the Bureau had been conducted. 